\title{
In Vitro Evaluation of the activity of Ceftazidime-avibactam and Aztreonam- Avibactam against 76 Stenotrophomonas maltophilia isolates from a teaching hospital in Chongqing
}

\section{Qiuxia Lin}

Chongqing Medical University First Affiliated Hospital

Hua Zou

Chongqing Health Center for Women and Children

Xian Chen

Affiliated Hospital of Medical College Qingdao University

Menglu Wu

Qingdao Women and Childrens Hospital

Deyu Ma

Chongqing Medical University First Affiliated Hospital

Hanbing Yu

Chongqing Medical University First Affiliated Hospital

Siqiang Niu

Chongqing Medical University First Affiliated Hospital

Shifeng Huang ( $\nabla$ sfhuang@hospital.cqmu.edu.cn)

1 Department of Clinical Laboratory Medicine, the First Affiliated Hospital of Chongqing Medical University https://orcid.org/0000-0001-7641-8823

\section{Research}

Keywords: Stenotrophomonas maltophiliam, ceftazidime-avibactam, aztreonam-avibactam, minimum inhibitory concentrations

Posted Date: August 3rd, 2020

DOI: https://doi.org/10.21203/rs.3.rs-45938/v1

License: (a) (i) This work is licensed under a Creative Commons Attribution 4.0 International License. Read Full License 


\section{Abstract}

Background: Treatment options for Stenotrophomonas maltophilia (S. maltophilia) infections were limited. We assessed the efficacy of ceftazidimeavibactam (CAZ-AVI) and aztreonam-avibactam (ATM-AVI) against a selection of 76 S. maltophilia out of the 1179 strains isolated from the First Affiliated Hospital of Chongqing Medical University during 2011-2018.

Methods: We investigated the antimicrobial resistance profiles of the 1179 S. maltophilia clinical isolates from the first affiliated hospital of Chongqing Medical University during 2011-2018, a collection of 76 isolates of which were available for further study of microbiological characterization. Minimum inhibitory concentrations (MICs) of ceftazidime (CAZ), ceftazidime-avibactam (CAZ-AVI), aztreonam (ATM) and aztreonam-avibactam (ATM-AVI) were determined via the broth microdilution method. We deemed that CAZ-AVI or ATM-AVI was more effective in vitro than CAZ or ATM alone when CAZ-AVI or ATM-AVI led to a category change from "Resistant" with CAZ or ATM alone to "Susceptible" or "Intermediate" with CAZ-AVI or ATM-AVI, or if the MIC of CAZAVI or ATM-AVI was at least 2-fold lower than the MIC of CAZ or ATM alone.

Results: For the 76 clinical isolates included in the study, MICs of CAZ, ATM, CAZ-AVI and ATM-AVI ranged from 0.03-64, 1-1024, 0.016-64, and 0.06-64 $\mu \mathrm{g} / \mathrm{mL}$, respectively. In combined therapy, AVI was effective at restoring the susceptibility of $48.48 \%(16 / 33)$ and $89.71 \%(61 / 68)$ of $S$. maltophilia to CAZ and ATM, respectively. Furthermore, CAZ-AVI showed better results in terms of the proportion of susceptible isolates $(77.63 \%$ vs.56.58\%, P<0.001), MIC50 $(2 \mu \mathrm{g} / \mathrm{mL}$ vs. $8 \mu \mathrm{g} / \mathrm{mL}, \mathrm{P}<0.05)$, and MIC distribution $(P<0.001)$ when compared to CAZ. According to our definition, CAZ-AVI was more effective in vitro than CAZ alone for $84.21 \%$ of the isolates. Similarly, ATM-AVI also showed better results in terms of the proportion of susceptible isolates ( $90.79 \%$ vs. $10.53 \%$, $\mathrm{P}<0.001)$, MIC50 $(2 \mu \mathrm{g} / \mathrm{mL}$ vs. $64 \mu \mathrm{g} / \mathrm{mL}, \mathrm{P}<0.001)$, and MIC distribution $(\mathrm{P}<0.001)$ when compared to ATM. According to our definition, ATM-AVI was also more effective in vitro than ATM alone for $97.37 \%$ of the isolates.

Conclusions: AVI potentiated the activity of both CAZ and ATM against S. maltophilia clinical isolates in vitro. We demonstrated that CAZ-AVI and ATM-AVI are both useful therapeutic options to treat infections caused by S. maltophilia.

\section{Introduction}

Stenotrophomonas maltophilia (S. maltophilia) is a Gram-negative, nonfermentative, environmental bacillus that has emerged as an important cause of nosocomial infections in immunocompromised hosts. In patients with cystic fibrosis (CF), S. maltophilia is known for colonizing the airways and causing chronic infections $[1,2]$. Although $S$. maltophilia is primarily associated with respiratory tract infections, this pathogen can cause a wide range of clinical syndromes, including catheter-associated bloodstream infections, and skin and soft tissue infections [2-4]. Furthermore, although S. maltophilia is not a highly virulent pathogen, it has emerged as an important nosocomial pathogen associated with crude mortality rates ranging from 14 to $69 \%$ in patients with bacteremia $[5,6]$. S.maltophilia is recognized by the World Health Organization as one of the leading MDR organisms in hospital settings for which disease prevention and treatment strategies must be developed [7]. More frustratingly, S. maltophilia is intrinsically resistant to different classes of antibiotics used in clinical practices, which was mediated by the expression of aminoglycoside-modifying enzymes, qnrB-like quinolone-resistant determinants, multidrug efflux pumps, and two $\beta$-lactamases (L1 and L2) $[1,8,9]$. These characteristics, together with its ability to adapt to environmental changes, contribute to the difficulty in effectively managing infections with $S$. maltophilia.

Ceftazidime, levofloxacin, minocycline, and trimethoprim-sulfamethoxazole have been used as treatment options for S.maltophilia infections; but unfortunately, their susceptibility against S.maltophilia is declining [10]. To assist clinicians in achieving effective individualized and precise treatment for S.maltophilia infections, more high-quality epidemiology- and antimicrobial susceptibility testing (AST) to novel antibiotics-centered studies are urgently needed. $\beta$-lactamases are enzymes that hydrolyze the $\beta$-lactam amide, inactivating them, and preventing them from reaching their target, the penicillin binding protein in bacterial cell membranes. S. maltophilia isolates naturally produce two $\beta$-lactamases (L1 and L2). L1 is a class B3 metallo- $\beta$-lactamase $(\mathrm{MBL})$ that hydrolyzes carbapenems and other $\beta$-lactams, with the important exception of the monobactam aztreonam (ATM), and is resistant to all clinically available $\beta$-lactams $[1,8,9]$. L2 is a class A cephalosporinase that confers resistance to extended-spectrum cephalosporins and ATM but can be inhibited by commercially available serine- $\beta$-lactamase inhibitors such as clavulanic acid and avibactam[1,2,11,12]. Avibactam (AVI) is a non $\beta$ lactam, $\beta$ lactamase inhibitor without intrinsic antibacterial activity, but offers a broader $\beta$-lactamase inhibition profile compared with any other recently used serine $\beta$ lactamase inhibitors [13]. Although S. maltophilia isolates naturally produce two $\beta$-lactamases (L1 and L2), the bactericidal activities of CAZ and ATM against L2-producing S. maltophilia isolates might theoretically be re-established by AVI combination; On the other side, given that the potential bactericidal activity of ATM against L1-producing S. maltophilia isolates might theoretically be achieved by monobactam's activity against MBL-producers, when combined with AVI, ATM/AVI might be useful to treat infections with both L1- and L2-producers, thus, AVI might restore the activity of ATM against both L1 and L2-producing S. maltophilia clinical isolates in vitro. Nevertheless, two recent studies from Suresnes and Japan demonstrated that CAZ-AVI is not active against $S$. maltophilia [14,15]. However, another report from France showed that while one third of the $S$. maltophilia isolates studied remained resistant to CAZ-AVI, 30\% showed low MICs ( $<1 \mathrm{ug} / \mathrm{mL})$ [16], highlighting the potential benefit of CAZ-AVI against S. maltophilia. Therefore, for better decision making of the clinical management of $S$. maltophilia infections, additional epidemiology and resistance testing of $S$. maltophilia isolates from other countries or regions worldwide are desperately needed. In addition, alternative therapeutic strategy for $S$. maltophilia is urgently needed. So far, there are few data available in China describing the in vitro activities of CAZ-AVI and ATM-AVI against $S$. maltophilia clinical isolates. We conducted an observational study to evaluate the in vitro antimicrobial activities of CAZ/AVI and ATM/AVI against recent $S$. maltophilia clinical isolates in our hospital. In this paper, we demonstrated that CAZ-AVI combination inhibited nearly half $(16 / 33,48.48 \%)$ CAZ-non-susceptible $S$. maltophilia isolates, while ATM-AVI combination inhibited most $(61 / 68,89.71 \%)$ of the ATM-non-susceptible $S$. maltophilia strains.

\section{Materials And Methods}




\section{Bacterial strains}

We collected a total of 76 non-repetitive, recent nosocomial S. maltophilia strains between 2014 and 2018 in the First Affiliated Hospital of Chongqing Medical University. All the isolates were identified at the species level by the VITEK MS (bioMérieux, MO, USA) system, and routine antimicrobial susceptibility testing was performed using the disk diffusion (for levofloxacin, minocycline, and trimethoprim-sulfamethoxazole) testing methods. All the $S$. maltophilia colonization and infection cases (1179) with complete medical records during 2011-2018 were investigated for clinical and antimicrobial resistance profiles, among which 76 recent isolates during 2014-2018 were selected for further microbiological characterization.

\section{Antibiotics and in vitro antimicrobial susceptibility testing}

A collection of 76 non-repetitive S. maltophilia isolates during 2014-2018 were recovered for CAZ, CAZ/AVI, ATM, and ATM/AVI susceptibility tests. MIC values of CAZ, CAZ/AVI, ATM, and ATM/AVI were determined by broth microdilution method as described by the Clinical and Laboratory Standards Institute (CLSI) M07-Ed11 (2019) [17]. MICs of CAZ and ATM alone and in combination with AVI at a fixed concentration of $4 \mu \mathrm{g} / \mathrm{mL}$ were measured. Antibiotic solutions for susceptibility testing were prepared fresh. All samples were incubated at $35^{\circ} \mathrm{C}$ for 18 to $20 \mathrm{~h}$ prior to MIC determination [17]. MICs of CAZ, ATM and comparator agents were interpreted according to CLSI criteria in M100-Ed29, 2019. MICs of CAZ/AVI >=16/4 $\mu \mathrm{g} / \mathrm{mL}$ and ATM/AVI >=16/4 $\mu \mathrm{g} / \mathrm{mL}$ were considered resistant.

\section{Statistical analysis}

All analyses were performed using the SPSS v.25.0 software (SPSS Inc., IL, USA). For all calculations, P $<0.05$ was considered statistically significant.

\section{Results}

\section{Microbiological characteristics and antimicrobial susceptibility profiles of S. maltophilia isolates}

As shown in Figure 1, 1179 non-repetitive S. maltophilia strains were isolated during the study period, among which the predominant sample origins were sputum (73.0\%), followed by secretion (7.0\%) and urine (5.0\%). Department distribution analysis showed that ICU (23.0\%) including the respiratory intensive care unit (10.0\%), and the respiratory department (9.0\%), contributed the majority of S. maltophilia (Fig 2). With regard to the antimicrobial susceptibility profiles of the S. maltophilia isolates, its non-susceptible rates to levofloxacin, minocycline, and trimethoprim-sulfamethoxazole were respectively $9.08 \%$, $3.14 \%$, and $6.28 \%$ (Table 1$)$.

\section{Bactericidal activities of CAZ/AVI and ATM/AVI against S. maltophilia isolates}

To assess the potential efficacy of CAZ/AVI and ATM/AVI against S. maltophilia isolates, we have tested these combinations in vitro on a recent collection of 76 non-repetitive isolates available for microbiological characterization. The majority of tested isolates $(60 / 76,78.95 \%)$ were resistant to at least one of the following three antibiotics, namely levofloxacin (LVX), minocycline (MH), and trimethoprim-sulfamethoxazole (SXT). While the rest part of the isolates were sensitive to LVX, MH, and SXT. The in vitro antimicrobial susceptibilities of CAZ, CAZ/AVI, ATM, and ATM/AVI against these isolates were determined using the CLSI broth microdilution method. The MIC of CAZ alone was $<=8 \mu \mathrm{g} / \mathrm{mL}$ for 43 of $76 \mathrm{~S}$. maltophilia isolates (56.58\%), and the MIC of CAZ-AVI was $<=8 \mu \mathrm{g} / \mathrm{mL}$ for 59 of 76 isolates (77.63\%) (Table 2). For the 21 out of the 33 (63.64\%) S. maltophilia isolates that were CAZ-nonsusceptible, the combination of AVI restored 13 strains' susceptibility to CAZ, remaining 8 isolates resistant to CAZ-AVI (main MIC: $64 / 4 \mu \mathrm{g} / \mathrm{mL}$ ). AVI addition also reduced the CAZ MIC50 of the $76 \mathrm{~S}$. maltophilia isolates from 8 to $2 \mu \mathrm{g} / \mathrm{mL}$ (Table 3). In all, the MIC of ATM alone was $<=8 \mu \mathrm{g} / \mathrm{mL}$ for 8 of the $76 \mathrm{~S}$. maltophilia isolates (10.53\%), and the MIC of ATM-AVI was $<=8 \mu \mathrm{g} / \mathrm{mL}$ for 69 of 76 isolates (90.79\%) (Table 2). Notably, for 66 out of the 68 (97.06\%) S. maltophilia isolates that were ATM-nonsusceptible, the addition of AVI restored their susceptibility to ATM. AVI addition also reduced the ATM MIC50 of the $76 \mathrm{~S}$. maltophilia isolates from 64 to $2 \mu \mathrm{g} / \mathrm{mL}$ (Table 3 ).

The results of susceptibility testing comparing CAZ, CAZ-AVI, ATM and ATM-AVI, are shown in (Table 3).These agents showed a wide range of activity against S. maltophilia. In detail, the ranges of MICs of CAZ and ATM for the 76 clinical S. maltophilia isolates were 0.03 to 64 and 1 to $1024 \mu \mathrm{g} / \mathrm{mL}$, respectively, while those of CAZ-AVI and ATM-ACI for the same S. maltophilia isolates were 0.016 to 64 and 0.06 to $64 \mu \mathrm{g} / \mathrm{mL}$, respectively (Table 3 ). In this study, AVI potentiated the activity of ATM against most of the S. maltophilia clinical isolates tested in vitro. Meanwhile, AVI also enhanced the activity of CAZ against most of those isolates tested in vitro. On the one hand, for S. maltophilia isolates that were nonsusceptible to LVX, MH, and/or SXT (78.95\%, 60 of 76), the addition of $4 \mu \mathrm{g} / \mathrm{mL} \mathrm{AVI}$ greatly increased the activity of CAZ against most species (4-fold MIC50 reduction) and the addition of $4 \mu \mathrm{g} / \mathrm{mL}$ AVI also significantly increased the activity of ATM against most species (32-fold MIC50 reduction) (Table 3). On the other hand, AVI did not restore the activity of CAZ against the two multidrug-resistant (MDR) S. maltophilia isolates, even though AVI reduced one MDR isolate's MIC somewhat (4-fold MIC reduction) (Table 3). However, AVI did restore the activity of ATM against the two MDR S. maltophilia isolates with obvious MIC reduction of 32- or 8-fold. (Table 3)

\section{CAZ/AVI and ATM/AVI are more effective in vitro than CAZ and ATM alone against $S$. maltophilia isolates.}


When compared to CAZ, CAZ-AVI showed better results in terms of the proportion of susceptible isolates $((77.63 \% \mathrm{vs} .56 .58 \%, \mathrm{P}<0.001), \mathrm{MIC50}(2 \mu \mathrm{g} / \mathrm{mL}$ vs. $8 \mu \mathrm{g} / \mathrm{mL}, \mathrm{P}<0.05)$, and MIC distribution (Table 4) ( $\mathrm{P}<0.001)$. According to our definition, CAZ-AVI was more effective in vitro than CAZ alone for $84.21 \%$ of the isolates. On the other hand, ATM-AVI likewise showed better results in terms of the proportion of susceptible isolates ( $90.79 \%$ vs. $10.53 \%$, $P<0.001)$, MIC50 $(2 \mu \mathrm{g} / \mathrm{mL}$ vs. $64 \mu \mathrm{g} / \mathrm{mL}, \mathrm{P}<0.001)$, and MIC distribution (Table 5) $(P<0.001)$ when compared to ATM. According to our definition, ATM-AVI was also more effective in vitro than ATM alone for $97.37 \%$ of the isolates

\section{Discussion}

S. maltophilia infections pose a major challenge for clinicians because of limited therapeutic options. For the 76 clinical isolates included in the present study, both CAZ-AVI and ATM-AVI exerted promising results in terms of the proportion of susceptible isolates, MIC50, and MIC distribution. Furthermore, while ATM-AVI was more effective in vitro than ATM alone for $97.37 \%$ of the isolates, CAZ-AVI was more effective in vitro than CAZ alone for $84.21 \%$ of the isolates. However, it is noteworthy that CAZ-AVI resistance was found in $17 \mathrm{~S}$. maltophilia strains isolated from patients with no history of previous CAZ-AVIbased treatment, moreover, $7 \mathrm{~S}$. maltophilia strains isolated from patients without previous ATM-AVI exposure demonstrated in vitro resistance to ATM-AVI, indicating that incidence of CAZ-AVI and ATM-AVI resistance could emerge in S. maltophilia strains without previous antimicrobial exposure. Notably, although AVI did not restore the activity of CAZ against the 2 MDR S. maltophilia isolates, it did restore the activity of ATM against the two MDR $S$. maltophilia strains with significant MIC reductions of 32- and 8-fold respectively. The poor activity of CAZ-AVI against MDR S. maltophilia isolates was in accordance with a previous study by Lindsay J. Caverly et al., who demonstrated that the activity of CAZ-AVI was poor against most MDR/XDR $S$. maltophilia strains. Recently, the efficacy of CAZ-AVI ( $2.5 \mathrm{~g}$ i.v. every $8 \mathrm{~h})$ in combination with ATM ( $2 \mathrm{~g}$ i.v. every $8 \mathrm{~h}$ ) for 48 days was demonstrated for a young renal transplant patient with $S$. maltophilia resistant to SXT, meropenem and CAZ [11]. In our research, CAZ/AVI has been demonstrated to inhibit the growth of about half of the CAZ-NS isolates $(48.48 \%, 16 / 33)$, still showing high-level resistance (MIC: $64 / 4 \mu \mathrm{g} / \mathrm{mL}$ ) in 10 isolates. Nevertheless, compared with CAZ/AVI, ATM/AVI exhibited obviously superior bactericidal activity, inhibiting the growth of $89.71 \%$ of the ATM-NS isolates (61/68) (Table 2 and Figure $3)$. The synergy among CAZ, ATM and AVI is encouraging and deserves further exploration. In this study, we likewise confirmed the emergence of both CAZAVI-resistant and ATM-AVI-resistant S. maltophilia strains isolated from patients without previous antimicrobial exposure to CAZ-AVI and ATM-AVI, which are consistent with our previously findings that demonstrated CAZ-AVI resistance in the carbapenem-resistant Enterobacteriaceae (CRE) bacteremia isolates from patients with no history of previous CAZ-AVI exposure [18].

The current study has several limitations. First of all, we only evaluated the activity of CAZ, ATM, CAZ-AVI and ATM-AVI without exploring the resistance mechanisms for both CAZ-AVI and ATM-AVI non-susceptibilities in our S. maltophilia isolates. Secondly, this was a single-center retrospective study with relatively small sample size conducted in Chongqing.

In summary, ATM-AVI showed the most potent in vitro activity among the other related agents, including CAZ, ATM and CAZ-AVI, against S. maltophilia isolates. The excellent in vitro activity of CAZ-AVI or ATM-AVI against $S$. maltophilia isolates in our hospital supports further evaluation of CAZ-AVI or ATMAVI in clinical studies against $S$. maltophilia infections. CAZ-AVI or ATM-AVI might turn out to be useful therapeutic options to treat infections caused by $S$. maltophilia.

\section{Abbreviations}

S. maltophilia: Stenotrophomonas maltophilia; CAZ-AVI: ceftazidime-avibactam; ATM-AVI: aztreonam-avibactam; MICs: Minimum inhibitory concentrations; CAZ: ceftazidime; ATM: aztreonam. Lvx: levofloxacin; MH: minocycline; SXT: trimethoprim-sulfamethoxazole.

\section{Declarations}

\section{Acknowledgments}

All authors read and approved the final manuscript.

\section{Author's contributions}

All authors contributed to data analysis, drafting and revising the article, gave final approval of the version to be published, and agree to be accountable for all aspects of the work.

\section{Funding}

This study was supported in part by the National Natural Science Foundation of China (Grant No. 81772239 and 31500749), the Science and Technology Research Program of Chongqing Municipal Education Commission (Grant No. KJ1500235 and KJ1702022), and the Medical Research Program of Chongqing Health and Family Planning Commission (No. 2018MSXM009 and 2016MSXM001).

\section{Availability of data and materials}

Not applicable. 


\section{Ethics approval}

The data and samples analyzed in the present study were obtained in accordance with the standards and approved by the Chongqing Medical University Institutional Review Board and Biomedical Ethics Committee. For this study, samples were collected at the microbiology laboratory of our hospital, with no contact with the patients. This study was retrospective and there was no patient identification performed during data collection. Therefore, the ethics committee determined that informed consent was not required.

\section{Consent for publication}

Not applicable.

\section{Competing interests}

The authors declare that they have no competing interests.

\section{References}

1. Brooke JS. Stenotrophomonas maltophilia: an emerging global opportunistic pathogen. Clin Microbiol Rev. 2012, 25, 2-41. DOI: 10.1128/CMR.0001911.

2. Chang YT, Lin CY, Chen YH, Hsueh PR. Update on infections caused by Stenotrophomonas maltophilia with particular attention to resistance mechanisms and therapeutic options. Front Microbiol. 2015, 6:893.http://dx.doi.org/10.3389/fmicb.2015.00893.

3. Looney WJ, Narita M, Mühlemann K. Stenotrophomonas maltophilia: an emerging opportunist human pathogen. Lancet Infect Dis. 2009 , 9, $312-323$. https://doi.org/10.1016/S1473-3099(09)70083-0.

4. Falagas ME, Kastoris AC, Vouloumanou EK, Dimopoulos G. Community-acquired Stenotrophomonas maltophilia infections: a systematic review. Eur J Clin Microbiol Infect Dis. 2009, 28, 719-730. https://doi.org/10.1007/s10096-009-0709-5.

5. Jang TN, Wang FD, Wang LS, Liu CY, Liu IM. Xanthomonas maltophilia bacteremia: an analysis of 32 cases. J. Formos. Med. Assoc. 1992, 91, 11701176.

6. Verweij PE, et al. Nosocomial outbreak of colonization and infection with Stenotrophomonas maltophilia in preterm infants associated with contaminated tap water. Epidemiol Infect. 1998, 120,251-256.

7. World Health Organization. 2018. Public health importance of antimicrobial resistance. https://www.who.int/drugresistance/AMR_Importance/en/

8. Crossman LC, Gould VC, Dow JM, Vernikos GS, Okazaki A, Sebaihia M, Saunders D, Arrowsmith C, Carver T, Peters N, Adlem E, Herhornou A, Lord A, Murphy L, Seeger K, Squares R, Rutter S, Quail MA, Rajandream MA, Harris D, Churcher C, Bentley SD, Parkhill J, Thomson NR, Avison MB. The complete genome, comparative and functional analysis of Stenotrophomonas maltophilia reveals an organism heavily shielded by drug resistance determinants. Genome Biol. 2008, 9, R74. https://doi.org/10.1186/gb-2008-9-4-r74.

9. Okazaki A, Avison MB. Induction of L1 and L2 $\beta$-lactamase production in Stenotrophomonas maltophilia is dependent on an AmpR-type regulator. Antimicrob Agents Chemother. 2008, 52, 1525-1528. https://doi.org/10.1128/AAC.01485-07.

10. Sader HS, Farrell DJ, Flamm RK, Jones RN. Antimicrobial susceptibility of Gram-negative organisms isolated from patients hospitalized with pneumonia in US and European hospitals: results from the SENTRY antimicrobial surveillance program, 2009-2012. Int J Antimicrob Agents. 2014, 43, 328-34.

11. Mojica MF, Papp-Wallace KM, Taracila MA, Barnes MD, Rutter JD, Jacobs MR, LiPuma JJ, Walsh TJ, Vila AJ, Bonomo RA. Avibactam restores the susceptibility of aztreonam against clinical isolates of Stenotrophomonas maltophilia. Antimicrob Agents Chemother. 2017, 61(10), e00777-

17. https://doi.org/10.1128/AAC.00777-17.

12. Mojica MF, Ouellette CP, Leber A, Becknell MB, Ardura MI, Perez F, Shimamura M, Bonomo RA, Aitken SL, Shelburne SA. Successful treatment of bloodstream infection due to metallo-beta-lactamase-producing Stenotrophomonas maltophilia in a renal transplant patient. Antimicrob Agents Chemother. 2016, 60, 5130-5134. https://doi.org/10.1128/AAC.00264-16.

13. Wong D, van Duin D. Novel beta-lactamase inhibitors: unlocking their potential in therapy. Drugs 2017, 77(6), 615-628.

14. Yohei Doi. Treatment Options for Carbapenem-resistant Gram-negative Bacterial Infections. Clinical Infectious Diseases. 2019,69 (Suppl 7), S565

15. Farfour E, Trochu E, Devin C, et al. Trends in ceftazidime-avibactam activity against multidrug-resistant organisms recovered from respiratory samples of cystic fibrosis patients. Transplant Infectious Disease. 2018, e12955

16. Moriceau C, et al. Stenotrophomonas maltophilia susceptibility to ceftazidime-avibactam Combination versus ceftazidime alone. Med Mal Infect. 2020, https://doi.org/10.1016/j.medmal.2020.01.003

17. Clinical and Laboratory Standards Institute. Methods for dilution antimicrobial susceptibility tests for bacteria that grow aerobically; approved standard M07, 11th ed. Clinical and Laboratory Standards Institute, Wayne, PA. 2018.

18. Zou H, Xiong SJ, Lin QX, Wu ML, Niu SQ, Huang SF. CP-CRE/non-CP-CRE stratification and CRE resistance mechanism determination help in better managing CRE bacteremia using ceftazidime-avibactam and aztreonam-avibactam. Infect Drug Resist. 2019, 12, $3017-3027$.

\section{Tables}

Page 5/8 
Table 1. Antimicrobial susceptibility test results of 1179 S.maltophilia clinical isolates.

\begin{tabular}{|llclc|}
\hline Antibiotics & Sensitive & Intermediate & Resistant & Non-susceptible rate \\
\hline Levofloxacin (LVX) & 1082 & 26 & 82 & $9.08 \%$ \\
\hline Minocyline (MH) & 1142 & 25 & 12 & $3.14 \%$ \\
\hline Sulfamethoxazole (SXT) & 1105 & 21 & 53 & $6.28 \%$ \\
\hline & & & & \\
\hline
\end{tabular}

Table 2. MICs of CAZ and ATM in 76 S. maltophilia isolates: alone or in combination with $4 \mu \mathrm{g} / \mathrm{mL}$ AVI

\begin{tabular}{|lllllllllllllllllll|}
\hline antibiotics & \multicolumn{2}{l}{$\mathrm{MIC}(\mu \mathrm{g} / \mathrm{mL})$} & & & & & & & & & & & & & \\
& 0.016 & 0.03 & 0.06 & 0.12 & 0.25 & 0.5 & 1 & 2 & 4 & 8 & 16 & 32 & 64 & 128 & 256 & 512 & 1024 \\
\hline ATM & 0 & 0 & 0 & 0 & 0 & 0 & 1 & 1 & 1 & 5 & 1 & 2 & 59 & 2 & 0 & 3 & 1 \\
ATM+AVI & 0 & 0 & 1 & 2 & 0 & 4 & 18 & 35 & 5 & 4 & 2 & 3 & 2 & 0 & 0 & 0 & 0 \\
\hline CAZ & 0 & 1 & 0 & 0 & 0 & 1 & 3 & 8 & 12 & 18 & 9 & 2 & 22 & 0 & 0 & 0 & 0 \\
CAZ+AVI & 1 & 0 & 0 & 1 & 2 & 3 & 8 & 28 & 9 & 7 & 5 & 2 & 10 & 0 & 0 & 0 & 0 \\
\hline
\end{tabular}

Table 3. MIC50s, MIC90s, and ranges of MICs of CAZ-AVI and ATM-AVI for 76 S. maltophilia isolates from clinical specimens ${ }^{\mathrm{a}}$

\begin{tabular}{|c|c|c|c|c|c|c|c|c|c|c|c|c|c|c|}
\hline \multirow{2}{*}{$\begin{array}{l}\text { Drug } \\
\text { resistance } \\
\text { phenotype } \\
\text { (s) }{ }^{\text {b }} \\
\text { (no. of } \\
\text { isolates) }\end{array}$} & \multicolumn{3}{|l|}{ CAZ } & \multicolumn{3}{|c|}{$\mathrm{CAZ}+\mathrm{AVI}$} & \multirow{2}{*}{$\begin{array}{l}\text { CAZ-AVI } \\
\text { MIC50 } \\
\text { reduction } \\
\text { (fold) }\end{array}$} & \multicolumn{3}{|l|}{ ATM } & \multicolumn{3}{|c|}{ ATM+AVI } & \multirow{2}{*}{$\begin{array}{l}\text { ATM-AVI } \\
\text { MIC50 } \\
\text { reductior } \\
\text { (fold) }\end{array}$} \\
\hline & MIC50 & MIC90 & range & MIC50 & MIC90 & range & & MIC50 & MIC90 & range & MIC50 & MIC90 & range & \\
\hline Total $876 \rrbracket$ & 8 & 64 & $\begin{array}{l}0.03- \\
64\end{array}$ & 2 & 64 & $\begin{array}{l}0.016- \\
64\end{array}$ & 4 & 64 & 64 & $\begin{array}{l}1- \\
1024\end{array}$ & 2 & 8 & $\begin{array}{l}0.06- \\
64\end{array}$ & 32 \\
\hline $\begin{array}{l}\text { MH-NSC or } \\
\text { LVX- } \\
\text { NS } \\
\text { or SXT-NS } \\
(60)\end{array}$ & 16 & 64 & $\begin{array}{l}0.03- \\
64\end{array}$ & 4 & 16 & $\begin{array}{l}0.016- \\
64\end{array}$ & 4 & 64 & 64 & $\begin{array}{l}1- \\
1024\end{array}$ & 2 & 8 & $\begin{array}{l}0.06- \\
64\end{array}$ & 32 \\
\hline $\begin{array}{l}\mathrm{MH}-\mathrm{S}^{\mathrm{d}} \\
\text { and LEV-S } \\
\text { and SXT-S } \\
\text { (16) }\end{array}$ & 4 & 8 & $1-16$ & 2 & 2 & $0.25-8$ & 4 & 64 & 64 & 64 & 2 & 2 & $\begin{array}{l}0.5- \\
64\end{array}$ & 32 \\
\hline MDR (2) & $N A^{e}$ & NA & $>=64$ & NA & NA & $\begin{array}{l}16- \\
>64\end{array}$ & NA & NA & NA & 64 & NA & NA & $2-8$ & NA \\
\hline
\end{tabular}

${ }^{a}$ MICs are expressed in $\mu \mathrm{g} / \mathrm{mL} .{ }^{\mathrm{b}} \mathrm{CAZ}$, ceftazidime; ATM, aztreonam; AVI, avibactam; MDR, multidrug resistant. Multidrug-resistant isolates were defined as isolates demonstrating resistance to at least one antimicrobial agent from three or more different classes.

${ }^{c}$ NS, not susceptible. ${ }^{d}$ S, susceptible. e NA, not applicable. MIC50s and MIC90s are not presented for groups of fewer than 6 isolates.

Table 4. Comparison of Distributions of MICs of 76 S. maltophilia isolates to CAZ-AVI and CAZ 


\begin{tabular}{|c|c|c|c|c|c|c|c|c|}
\hline \multirow[t]{2}{*}{ Strains } & \multicolumn{3}{|c|}{ Group without AVI } & \multicolumn{3}{|c|}{ Group with AVI } & \multirow[t]{2}{*}{ Z } & \multirow[t]{2}{*}{$\mathbf{P}$} \\
\hline & $25^{\text {th }}(\mathrm{MIC})$ & $50^{\text {th }}(\mathrm{MIC})$ & $75^{\text {th }}(\mathrm{MIC})$ & $25^{\text {th }}(\mathrm{MIC})$ & $50^{\text {th }}(\mathrm{MIC})$ & $75^{\text {th }}(\mathrm{MIC})$ & & \\
\hline Total PMA isolates (76) & 4 & 8 & 64 & 2 & 2 & 8 & -4.484 & 0.000 \\
\hline CAZ resistance group ${ }^{a}(60)$ & 4 & 16 & 64 & 2 & 4 & 16 & -3.640 & 0.000 \\
\hline CAZ sensitive group ${ }^{b}(16)$ & 4 & 4 & 8 & 1 & 2 & 2 & -3.491 & 0.001 \\
\hline
\end{tabular}

a S.maltophilia isolates that are LVX-NS or MH-NS or SXT-NS (NS, not susceptible)

${ }^{b}$ S.maltophilia isolates that are LVX-, MH- and SXT-susceptible

Table 5. Comparison of Distributions of MICs of $76 \mathrm{~S}$. maltophilia isolates to ATM-AVI and ATM

\begin{tabular}{|c|c|c|c|c|c|c|c|c|}
\hline \multirow[t]{2}{*}{ Strains } & \multicolumn{3}{|c|}{ Group without AVI } & \multicolumn{3}{|c|}{ Group with AVI } & \multirow[t]{2}{*}{$\mathbf{Z}$} & \multirow[t]{2}{*}{$\mathbf{P}$} \\
\hline & $25^{\text {th }}(\mathrm{MIC})$ & $50^{\text {th }}(\mathrm{MIC})$ & $75^{\text {th }}(\mathrm{MIC})$ & $25^{\text {th }}(\mathrm{MIC})$ & $50^{\text {th }}(\mathrm{MIC})$ & $75^{\text {th }}(\mathrm{MIC})$ & & \\
\hline Total PMA isolates (76) & 64 & 64 & 64 & 1 & 2 & 2 & -10.216 & 0.000 \\
\hline ATM resistance group ${ }^{a}(60)$ & 64 & 64 & 64 & 1 & 2 & 2 & -8.947 & 0.000 \\
\hline ATM sensitive group ${ }^{\mathrm{b}}(16)$ & 64 & 64 & 64 & 1 & 2 & 2 & -4.960 & 0.000 \\
\hline
\end{tabular}

a S.maltophilia isolates that are LVX-NS or MH-NS or SXT-NS (NS, not susceptible)

${ }^{\mathrm{b}}$ S. maltophilia isolates that are LVX-, MH- and SXT-susceptible

\section{Figures}

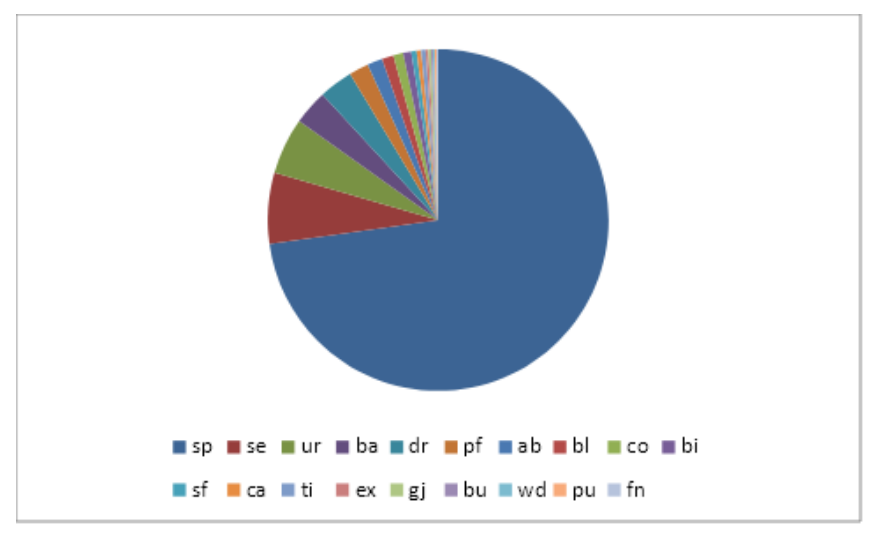

\section{Figure 1}

Specimen distribution of the 1179 S.maltophilia isolates sp: sputum; se: secretion; ur: urine; ba: broncho-alveolar lavage; dr: drawing fluid; pf: pancreatic drainage fluid; ab: abdominal fluid; bl: blood; co: concretion; bi: bile; sf: cerebrospinal fluid; ca: catheter; ti: tissue; ex: exudate; gj: gastric juice; bu: bursa; wd: wound secretion; pu: pus; fn: puncture fluid.

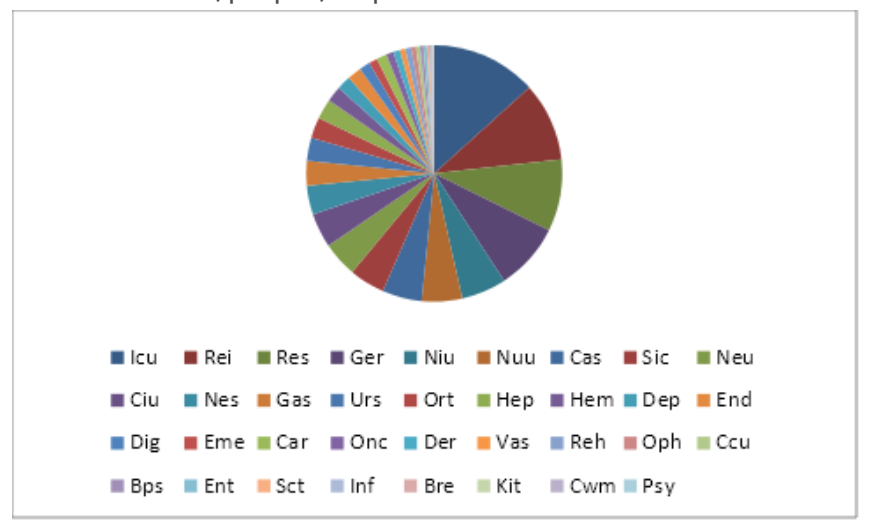


Figure 2

Ward distribution of the 1179 S.maltophilia isolates Icu: Intensive Care Unit; Rei: Respiratory Intensive Care Unit; Res: Respiratory Department; Ger:

Geriatrics Department; Niu: Neurology Intensive Care Unit; Nuu: Neurosurgery Intensive Care Unit; Cas: Cardiac Surgery Department; Sic: Surgery Intensive Care Department; Neu: Neurology department; Ciu: Cardiothoracic surgery; Nes: Neurosurgery Department; Gas: Gastrointestinal Surgery Department; Urs: Urology Surgery Department ; Ort: Orthopedics; Hep: Hepatological Surgery Department; Hem: Hematology Department; Dep: Department of Nephrology ; End: Endocrinology Department; Dig: Digestive System Department ; Eme: Emergency Department; Car: Cardiology Department; Onc: Oncology department; Der: Dermatology Department; Vas: Vascular Surgery Department; Reh: Rehabilitation Medicine department; Oph: Ophthalmology Department; Ccu: Cardiac Care Unit; Bps: Burn and Plastic Surgery Department; Ent: Ear, nose and throat specialist; Sct: Stem cell transplantation Center; Inf: Infections Department; Bre: Endocrine breast Surgery; Kit: Kidney Transplantation Ward; Cwm: Department of Integrated Chinese and Western Medicine; Psy: Psychiatry Department. 